\title{
Recent Trends in the Management of Graves' Hyperthyroidism in Japan: Opinion Survey Results, Especially on the Combination Therapy of Antithyroid Drug and Thyroid Hormone
}

\author{
TORU MORI, HIDEO SUGAWA, SHINJI KOSUGI, MichIKo UEDA, \\ NORITAKA HAI, AND AKIRA MATSUDA \\ Department of Laboratory Medicine, Kyoto University School of Medicine, Kyoto 606-01, Japan
}

\begin{abstract}
An opinion survey concerning the management of Graves' hyperthyroidism was conducted among the council members of the Japan Thyroid Association. The selection of 3 major treatments by 90 respondents for their patients was $98.6 \pm 4.2 \%$ for antithyroid drug (ATD), $7.8 \pm 12.6 \%$ for partial thyroidectomy and $5.2 \pm 8.1 \%$ for radioiodide. They expressed a movement away from the past trend of surgery because of postoperative complications and unsatisfactory therapeutic results, and they assumed a further reduction in the future. On the other hand, the frequency of radioiodide treatment was not considered to have decreased greatly, and they expected a slight increase in the future. Of the respondents, $65 \%$ suggested that hyperthyroidism should be completely cured even if the patient would fall into hypothyroidism. The major reascns for choosing surgery or radioiodide after ATD were the adverse effects of ATD and the age and social backgrounds of the patients. Large goiter size was the 3rd reason for surgery but was a minimal indicator for radioiodide. As for ATD treatment, none of the respondents reported the routine application of any uniform fixed-time therapy protocol. Japanese Graves' patients were shown to be less responsive to ATD than Caucasian patients. This was assumed to result at least from high iodide intake, and half of them had ordered their patients to restrict iodide intake. Furthermore, $78 \%$ of them had treated with a combined therapy of ATD and thyroid hormone. Most of them apply this for selected patients mainly to lower TSH receptor antibody activity, to better control their patients and to reduce the goiter size. All but $8\left(9 \%\right.$ ) did not give $\mathrm{T}_{4}$ (or $\mathrm{T}_{3}$ ) after the cessation of ATD, and they felt this to be unnecessary, doubtful about the effect, unsuitable or even possible to induce recurrence. The excellent findings reported by Hashizume et al. ( $N$ Engl J Med 324: 947-953, 1991) are well known among them. However, most of them did not agree with the efficacy of the protocol to reduce TRAb or to improve the remission rate, and $90 \%$ of the respondents did not intend to apply the protocol immediately. In conclusion, the Japanese thyroidologists were shown to highly prefer ATD, and they intended to treat their patients for longer periods of time only by ATD until clinical remission is achieved. The combination therapy is widely used, but most of them do not consider it effective. The therapeutic protocol reported by Hashizume et al. was not accepted widely in Japan.
\end{abstract}

Key words: Graves' disease, Antithyroid drug, TSH receptor antibody (TRAb), Clinical remission, Partial thyroidectomy, Radioiodide treatment

(Endocrine Journal 44: 509-517, 1997)

Received: January 20, 1997

Accepted: April 18, 1997

Correspondence to: Dr. Hideo SUGAWA, Department of Laboratory Medicine, Kyoto University School of Medicine, 54 Shogoin-Kawaharacho, Sakyo-ku, Kyoto 60601, Japan
GRAVES' disease is now considered to be an autoimmune disease causatively involved with autoantibodies against the TSH receptor [1-5]. Until now, however, there has been no causative treatment, and 3 major therapies, antithyroid drug 
(ATD), radioiodide and partial thyroidectomy, are generally used.

Large-scale opinion surveys concerning the management of Graves' hyperthyroidism were performed from 1986 to 1988 among the members of the European Thyroid Association, the American and Latin American Thyroid Associations and the Japan Thyroid Association [6-8]. These results indicated that there is no uniform and definite therapy protocols, and selection of therapeutic means is arbitrarily based on the clinical findings of the individual patients as well as the environmental factors or races.

In 1991, Hashizume et al. [9] reported excellent therapeutic findings by the combination therapy of ATD and l-thyroxine $\left(\mathrm{T}_{4}\right)$ for 1.5 years followed by 3 year- $-\mathrm{T}_{4}$ treatment. They observed a marked disappearance rate of TSH receptor antibody (TRAb) and also an extremely low recurrence rate. Many investigators have introduced similar therapeutic protocols, but, except for a few supportive findings, most of them failed to show such favorable results [10-16]. Some who had disappointing findings even said that Hashizume's protocol may be useful only for Japanese Graves' patients because of their rather unique life-style, especially their extremely high iodide intake [14, 15].

To investigate such suspicions was considered to be the responsibility of the Japanese thyroidologists. Furthermore, to determine the recent trends of the management of Graves' hyperthyroidism in Japan, we conducted an opinion survey among the council members of the Japan Thyroid Association. This paper describes the results of the survey, including the rather negative evaluation of Hashizume's protocol among the Japanese thyroidologists.

\section{Methods}

A questionnaire asked for the recent trend of the management of Graves' hyperthyroidism, including changes over the past 10 to 20 years and future prospects with reasons, for the opinion of ATD treatment in relation with the combined ATD $+\mathrm{T}_{4}$ (or $\mathrm{T}_{3}$ ) therapy, and for the evaluation of Hashizume's protcol [9]. This was sent to 192 council members of the Japan Thyroid Association. Ninety of them $(49 \%)$ responded. Of the 90 respondents, 76 were physicians (internal medicine, laboratory medicine, and nuclear medicine or radiology), 11 were surgeons, and 3 were of other specialties (pediatrics, gynecology and basic medical science). They saw an average of $72.4 \pm$ 219.2 (S.D.) new Graves' patients and an overall 676.2 Graves' patients in one year. These figures appear to be significant enough to represent general considerations of Japanese thyroidologists.

\section{Results}

\section{Selection of 3 major treatments}

Figure 1 shows the ratios of 3 major treatments applied by the 90 respondents. The questionnaire asked them to give a percentage of the 3 major treatments, ATD, surgery and radioiodide. When

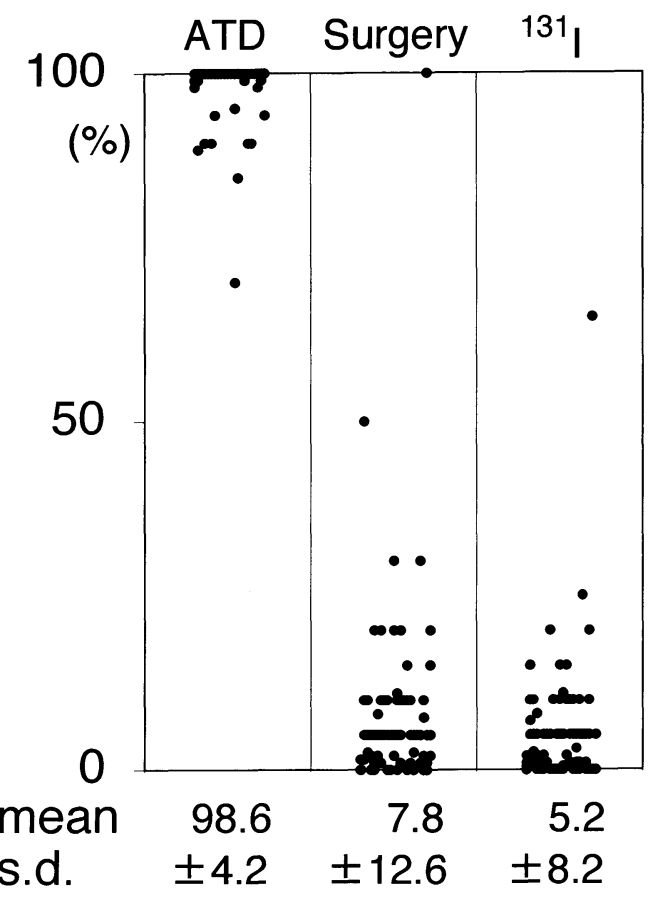

Fig. 1. Ratios of 3 major treatments for Graves' hyperthyroidism applied by the 90 respondents. The percentage given is not of the final treatment, but when previously ATD-treated patients were changed to another treatment later on, they were listed in both categories. Therefore, the sum of the percentages for 3 categories exceeds $100 \%$, but this does not affect the actual figures of surgery and radioiodide treatment. 
one patient was treated initially by ATD but then by another means, they were requested to report him (or her) in both categories. Therefore, the sum of the percentages exceeded $100 \%$. Though, the final calculations of the ratio of surgical or radioiodide treatment among all subjects were not affected. More than $70 \%$ of the patients were treated by ATD, giving an extremely high average value of $98.6 \pm 4.2 \%$. Surgical treatment was applied to $7.8 \pm 12.6 \%$ of the patients, and the highest given ratio was $100 \%$ by a surgeon. In the case of radioiodide, the highest value was $65 \%$, and the average was as low as $5.2 \pm 8.2 \%$.

Table 1 summarizes their present opinion compared to the past and future prospects of surgery and radioiodide treatments. As for the changes over the past 10 to 20 years, surgical treatment was indicated to have decreased greatly (46 of $84,55 \%)$, and only 5 respondents $(6 \%)$ answered some increases. In the case of radioiodide treatment, $32(38 \%)$ reported a decrease, 32 (39\%) stated no remarkable changes, and $19(23 \%)$ said it increased. As for future prospects, a further decrease in surgical treatment was expected $(17 \%)$. On the other hand, radioiodide treatment was predicted to increase (34\%) rather than decrease (12\%).

Among the reasons cited for the future prospect of surgical treatment, those who answered "will decrease" were mostly concerned about postoperative complications, unsatisfactory therapeutic results by surgery, and good therapeutic results by other treatments. Negative will of the patients and lack of excellent thyroid surgeons were also of minor concerns. As for radioiodide treatment, those who answered "will increase" greatly evaluated effectiveness and safety and were also concerned about the availability of a radioisotope facility and the patient's will. In contrast, a great concern of those who answered negatively for the future prospect of radioiodide treatment was a very high incidence of late onset hypothyroidism. Concerning late onset hypothyroidism, the respondents were asked for their therapeutic concepts of radioiodide treatment. As shown in Table 2, not only those who expected a future increase of radioiodide treatment (21 of 27, 78\%) but also those who predicted not much change in the future ( 28 of $42,67 \%$ ) and even some of the negative-outcome opinion ( 2 of $10,20 \%$ ) selected to achieve complete cure of hyperthyroidism even if the patient would fall into hypothyroidism (overall 51 of $79,65 \%$ ). To achieve euthyroidism was answered by 18 of 79 (23\%), and only 5 of 79 respondents $(6.3 \%)$ insisted in avoiding

Table 1. Therapeutic attitudes of the respondents for surgery and radioiodide at present compared to the past and their future prospects

1) Changes over the past $10-20$ years

\begin{tabular}{lcc}
\hline & Surgery & 131I \\
\hline increased & $1(1)$ & $3(4)$ \\
slightly increased & $4(5)$ & $16(19)$ \\
stationary & $33(39)$ & $32(39)$ \\
slightly decreased & $19(23)$ & $12(14)$ \\
decreased & $27(32)$ & $20(24)$ \\
\hline sum & $84(100)$ & $83(100)$ \\
\hline
\end{tabular}

2) Future prospects

\begin{tabular}{lcc}
\hline & Surgery & 131 I \\
\hline will increase & $2(2)$ & $28(34)$ \\
as it is & $68(80)$ & $45(54)$ \\
will decrease & $15(17)$ & $10(12)$ \\
\hline sum & $86(100)$ & $83(100)$ \\
\hline
\end{tabular}

Numbers in parentheses: percent. Sums in each category were different because of the number of answers given.

Table 2. Relationship between therapeutic concepts and future prospects for radioiodide 131I treatment

\begin{tabular}{lcccc}
\hline & will increase & as it is & will decrease & overall \\
\hline complete cure & 21 & 28 & 2 & 51 \\
euthyroidism & 4 & 11 & 3 & 18 \\
to avoid hypothyroidism & 2 & 3 & 5 & 10 \\
\hline \multirow{2}{*}{ sum } & 27 & 42 & 10 & 79 \\
\hline
\end{tabular}


hypothyroidism.

Almost all Graves' patients were treated by ATD initially. Table 3 summarizes the major reasons for choosing surgery or radioiodide after changing from ATD. Adverse effects of ATD and age or social backgrounds of the patients were the major concerns in both treatments. In the case of surgery, large goiter size was also a major concern but was a limited indication for radioiodide. Other reasons were poor sensitivity to ATD, irregular ATD intake (compliance), persistently high TRAb, relapsed cases and various complications.

\section{General considerations of ATD's effectiveness on Japanese Graves' patients}

None of the 90 respondents applied any uniform fixed-time ATD treatment protocol for their patients, but they treated patients individually and for a longer period of time.

When they were asked whether Japanese Graves' patients respond to ATD better than those in other countries, $10(12 \%)$ said "Yes" and 15 (18\%) said "No", but most of them $(58,70 \%)$ gave indefinite answers. Further, to the question, "Do ATD effects relate with the race?", 27 (33\%) responded "Yes", $10(12 \%)$ said "No", and $45(54 \%)$ gave no answer. The word "race" may include some environmental factors, especially food. Indeed, the Japanese are well known to have a very high iodide diet, more than $2 \mathrm{mg}$ daily. As shown in Table 4, high iodide intake was considered to be unfavorable for the ATD effects, and "No" answers $(36,40 \%)$ were more than twice of "Yes" $(17,19 \%)$. A half of them ordered their patients to restrict daily iodide intake, and only 1 of 88 respondents recommended a high iodide diet.

In summary, Japanese Graves' patients are considered to be less responsive to ATD probably because of their high iodide dietary habit, and they are mostly treated soley by ATD for a longer period of time compared to European or American patients.

Opinion of the combination therapy of $A T D+T_{4}$ (or $T_{3}$ ) and subsequent $T_{4}\left(\right.$ or $\left.T_{3}\right)$ administration

Figure 2 shows the practices of the combination therapy among the respondents. Only $8 \%$ of them applied the combination therapy routinely, and $58 \%$ applied this to selected patients. Further, $12 \%$
Table 3. Major reasons to choose surgery or $131 \mathrm{I}$ after changing from ATD

\begin{tabular}{lcc}
\hline & Surgery & 131 I \\
\hline adverse effects of ATD & 75 & 63 \\
age, social backgrounds & 51 & 46 \\
large goiter & 38 & 38 \\
poor sensitivity to ATD & 31 & 30 \\
irregular ATD intake & 25 & 20 \\
high TRAb & 14 & 16 \\
relapsed case & 10 & 13 \\
complications & 5 & 8 \\
\hline
\end{tabular}

Table 4. Considerations about the high iodide dietary intake on the effectiveness of ATD in Japanese Graves' patients

Japanese have a high iodide diet ......... $2 \mathrm{mg} /$ day or more.

Does high iodide intake induce a good response to ATD?

$\begin{array}{ll}\text { Yes } & 17 \\ \text { No } & 36 \\ \text { not definite } & 36\end{array}$

Do you order restrictions in iodide intake for your Graves' patients?

$\begin{array}{lc}\text { Yes } & 44 \\ \text { No } & 43 \\ \text { recommend high iodide } & 1\end{array}$

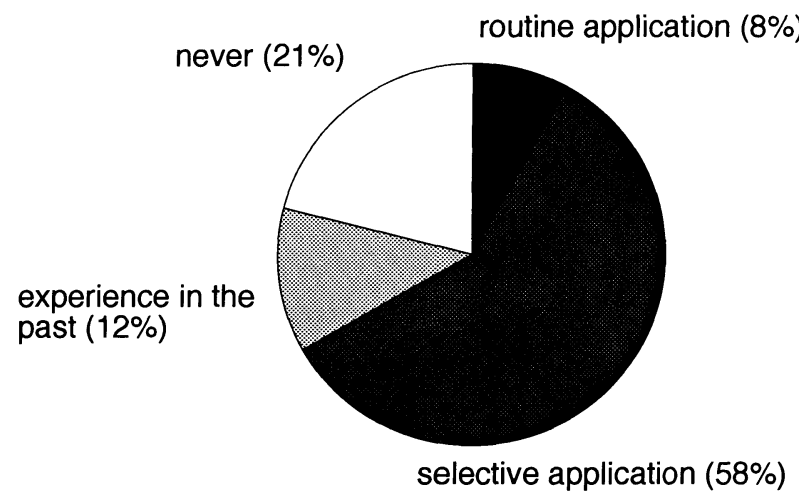

Fig. 2. Experience among the respondents of the combined therapy of ATD and $1-\mathrm{T}_{4}\left(\mathrm{~T}_{3}\right)$ on the management of Graves' hyperthyroidism.

of them had previous experience but are not applying it presently. The major reasons for selective application are shown in Table 5 . Persistently high TRAb (52\%), difficulty in controlling the patients only by ATD (44\%) and not enough reduction of goiter size by ATD (37\%) were the major concerns. Some $(8 \%)$ felt that 
Table 5. Major reasons for selective application of the combined therapy of ATD and 1- $\mathrm{T}_{4}$ (or $\mathrm{T}_{3}$ )

\begin{tabular}{lc}
\hline 1. TRAb is persistently high & $27(52)$ \\
2. hard to control with ATD only & $23(44)$ \\
3. goiter size is not reduced & $19(37)$ \\
4. association of ophthalmopathy & $4(8)$ \\
5. relapsed case & $1(2)$ \\
& $\%$ \\
\hline
\end{tabular}

Table 6. Opinions of the 90 respondents concerning the therapeutic protocol proposed by Hashizume et al. [9]

$\begin{array}{cc}\begin{array}{c}\text { Does this protocol improve the remission rate? } \\ \text { Yes }\end{array} & 11(12) \\ \text { No } & 50(56) \\ \text { not definite } & 29(32) \\ \% \\ \end{array}$

prevention of TSH elevation inducible during ATD treatment is desirable in cases with ophthalmopathy. The reasons why 11 respondents stopped applying the combination therapy were "not effective" (9), "unsuitable" (1), and "no reasons" (1).

Out of 87 respondents, only $8(9.2 \%)$ gave $\mathrm{T}_{4}$ (or $\mathrm{T}_{3}$ ) after the cessation of ATD. Unnecessary, doubtful about the effect, unsuitable or possibile recurrence induction were the major reasons why they did not give $T_{4}$ (or $T_{3}$ ). Even those who gave $\mathrm{T}_{4}$ (or $\mathrm{T}_{3}$ ) after ATD did so for a short period of time, usually less than 6 months.

Subsequently, they were asked to evaluate Hashizume's findings [9]. All but 1 respondents knew the paper (quite well $49 \%$, well $40 \%$ and fairly well $10 \%)$. As for the effectiveness of this protocol on reducing TRAb activities, 17 (19\%) said "Yes" but $37(41 \%)$ answered "No". Most of the respondents had given the combination therapy to reduce TRAb activities (Table 5), but they did not consider it to be very effective. As shown in Table 6, most of them (56\%) did not consider this protocol would improve the remission rate, and only $12 \%$ supported a possible improvement. Further, $48 \%$ of them will not apply this protocol
Table 7. Reasons given by the respondents for when they dicide to terminate ATD medication

\begin{tabular}{lcc}
\hline & present survey & 1988 survey [8] \\
\hline Disappearance of TRAb & $82(91)$ & $(77)$ \\
Reduction in goiter size & $49(54)$ & $(4)$ \\
Positive T3 suppression & $33(37)$ & $(47)$ \\
Elevation of serum TSH & $30(33)$ & $(59)$ \\
Miscellaneous* & $16(18)$ & \\
Duration of ATD treatment & $12(13)$ & $(58)$ \\
& $\%$ & $\%$ \\
\hline
\end{tabular}

*: mainly because of good control by the minimal ATD dosage.

in the future, and $42 \%$ will wait until acceptable findings are demonstrated by other institutions but not by themselves. Yet, 9 (11\%) of them answered to have been applying or to apply this protocol.

The final question asked for the reasons in deciding when to terminate ATD medication (Table 7). Disappearance of TRAb was the major reason, and reduction in goiter size, positive $T_{3}$ suppressibility and elevation of serum TSH followed. One considered good control by a minimal dosage of ATD or long duration of ATD treatment meaningful, and that decision should be made individually based on the combination of several reasons. Interestingly, however, frequency and ranking of the reasons in this survey were slightly different from those shown in the 1988 survey [8].

\section{Discussion}

There has been no universally accepted protocol for the management of Graves' hyperthyroidism. Through this survey, Japanese thyroidologists were found to prescribe ATD to almost all their patients and prefered to treat them for a longer period of time until clinical remission is achieved. In Europe, however, a shorter fixed-time ATD protocol is preferred and is resulting in a comparably high remission rate $[6,11,14,17-26]$. This may be partly related to the low iodide intake of Europeans. In contrast, a very high rate of application of radioiodide treatment has been indicated by American thyroidologists [7]. This may greatly depend on its cost effectiveness as well as good therapeutic results, and they are not concerned as much about the highly possible development of late onset hypothyroidism. Even in Japan, 
radioiodide treatment was widely applied around 1960 [21]. However, because of a very high incidence of late onset hypothyroidism and general negative considerations of patients for the acceptance of radioisotopes, usage of radioiodide has recently become less frequent.

The present survey revealed that the ratios of 3 major treatments applied were $98.6 \pm 4.2 \%$ for ATD, $7.8 \pm 12.6 \%$ for surgery and $5.2 \pm 8.1 \%$ for radioiodide. Though the style of the questionnaire is different, these figures show significant changes from those of the 1988 survey [8], in which a typical middle-aged case and its variables with Graves' disease were presented and asked for the choice of treatment, resulting in the respective figures of $75 \%$, $7 \%$ and $18 \%$. A marked decrease in radioiodide treatment was indicated; however, when the respondents were asked for their present opinion compared to that of 10 to 20 years ago, the replies of more than half of them showed a decrease in choosing surgery. In the case of radioiodide treatment, 38\% of them considered it "decreased" and 23\% "increased". Moreover, they considered a further decrease of surgery in the future because of 1) undesirable postoperative complications, such as hypoparathyroidism, reccurence palsy or ugly scars, 2) unsatisfactory therapeutic results by surgery, considerably high incidence of postoperative hypothyroidism and infrequent but problematic relapses, and 3) relatively good therapeutic results by other treatments. On the other hand, they gave a much higher evaluation of the future prospect for the radioiodide treatment mostly because of its effectiveness and safety. Of course, the very high incidence of late onset hypothyroidism was also of great concern. However, when asked for the therapeutic concepts about the radioiodide treatment, $65 \%$ of the respondents chose this treatment to achieve complete cure of hyperthyroidism even if the patients would fall into hypothyroidism later. They appeared to apply radioiodide to a limited number of patients as an emergency resolution necessary to eradicate persistent hyperthyroidism, which is associated with adverse effects of ATD, poor responsiveness to ATD or troublesome complications. Cost effectiveness, which was the major concern of the US thyroidologists, was not considered seriously, but Japan should also take this into account in the future.

The reasons for choosing surgery or radioiodide after changing from ATD were essentially the same, except for the concerns about goiter size. The biggest reason was the adverse effects by ATD, followed by the age or social background of the patients. In the latter, however, surgery is likely to be preferred for the younger, active patients and radioiodide for the aged ones. Large goiter size was the 3rd biggest concern in the case of surgery but was a minimal indication for radioiodide. The reasons for this are not clear, but it is assumed likely to be that ATD cannot induce remission quickly in patients with large goiters, $[12,18,19$, 22,23 ] and that young, socially active patients are more apt to eliminate troubles quickly, but most aged patients with large goiters accept the amelioration of hyperthyroidism by ATD and are not willing to accept other radical treatments.

The major purpose of the present survey was to see the opinion and evaluation of the effectiveness of the combination therapy of ATD and l-thyroxine and TSH suppressive treatment after the cessation of ATD among the Japanese thyroidologists. As for the ATD treatment protocol, none of the 90 respondents applied any uniform fixed-time protocol. This is greatly different from the survey findings for European thyroidologists [11, 14, 1720]. In general, Japanese patients with Graves' disease are considered to be less responsive to ATD and need to be treated longer [21]. Both racial and environmental factors may relate to such a difference. Genetic factor(s) critically related to the inheritance of Graves' disease has not been clarified yet. However, associations of HLA antigens have been known to be greatly different between the Japanese and Caucasian patients [4, 5]. Regarding the autoantibody-involved autoimmune nature of the disease, associations of HLA class II antigens, T cell epitope analysis, TCR clonalities as well as immunoglobulin gene analysis for TSH receptor antibodies have been reported [5]. There are, at least, apparent differences in the disease-associated class II antigens. Graves' disease may only be a generic term for similar clinical manifestations, but it may consist of genetically and phenotypically different subtypes among races or even individual patients $[5,24]$. One of the most characteristic environmental factors different from Japan and Europe is the quantity of the daily iodide intake. A high iodide diet was demonstrated to induce frequent relapse and to promote thyroidrelated immune responses [14, 25-27]. Many of 
the respondents considered high iodide intake unfavorable for ATD-treated Japanese Graves' patients, and half of them ordered dietary iodide restrictions for their patients.

The combination therapy of ATD and thyroid hormone $\left(T_{4}\right.$ or $\left.T_{3}\right)$ has been applied for many years, but the superiority of this over ATD alone had not been clearly addressed until the excellent findings were reported by Hashizume et al. [9] in 1991. One significant finding they observed was the very rapid and effective reduction in TRAb in the combination therapy group patients compared to the placebo and ATD group. Since then, many thyroidologists applied similar combination therapies but most of them, including Tamai et al. [13] for Japanese patients, were not able to confirm the superiority of the combination therapy in TRAb reduction [10, 11, 13-15]. Kuo et al. [16] only reported supportive results. Among the present respondents, $78 \%$ answered to practice or have practiced the combination therapy. Generally, they applied it to selected patients with 1) persistently high TRAb, 2) difficultly in controlling only by ATD, and 3) large goiter. However, they gave rather negative opinions of the combination therapy concerning the reduction in TRAb. ATD is effective in ameliorating hyperthyroidism in almost all Graves' patients. However, clinical remission cannot be expected in patients with high TRAb and/or large goiter. To facilitate the clinical remission, a higher dose of ATD than that is necessary to maintain euthyroid states. Increase of ATD dosage should induce hypothyroidism, TSH elevations, then enlargement of goiter. Therefore, it is necessary to give $1-T_{4}$ or $T_{3}$ together with ATD. This may reduce TRAb to some extent, and some respondents favored this. The

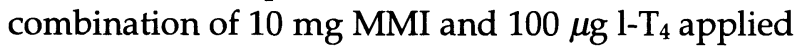
by Hashizume et al. [9] may be considered insufficient by many of the respondents.

As for the 3-year post-ATD treatment of $1-\mathrm{T}_{4}$ (or $T_{3}$ ), most of the respondents did not support its usefulness in improving the remission rate but considered it to be unnecessary, ineffective, unsuitable or even possible to induce recurrence. Only 8 of them gave $1-T_{4}$ or $T_{3}$ to their patients but mostly for a short period of time, 6 months or less.
Overall, the respondents do not favor the Hashizume protocol; only $11 \%$ of them would apply this in the future, $48 \%$ had no intention, and the rest would consider it after seeing the results of other institutions but not of their own. Therefore, 90 council members of the Japan Thyroid Association do not consider the Hashizume protocol to be exceptionally advantageous or suitable for Japanese Graves' patients.

As mentioned, a great many of the Japanese Graves' patients are treated only by ATD or a combination with $1-\mathrm{T}_{4}$ for a longer period of time. Then, to decide if clinical remission has been achieved, disappearance of TRAb, reduction of goiter size, positive $T_{3}$ suppressibility, elevation of serum $\mathrm{TSH}$, and long lasting euthyroid state under a minimal ATD dose were chosen in this order as the criteria. The order is slightly different from that reported in the 1988 survey [8] which indicated the goiter size much less significant. We considered the reduction of goiter size in long ATDtreated patients to be important for the achievement of clinical remission. Recently, we have reported the effectiveness of short-term steroid administration in such patients to induce goiter reduction and clinical remission further [23].

Through this survey, we could not show the remission rate by ATD treatment in Japan due to unclear wording of the questionnaire. We have realized again that there is no definite, effective management of Graves' hyperthyroidism applied universally. An investigation of a new causatively related immune suppressive therapy is needed in the near future.

\section{Acknowledgements}

The authors would like to express their sincere gratitude to the 90 respondents among the council members of the Japan Thyroid Association for kindly answering the questionnaire, to Miss A. Nakajima and E. Ito for secretarial assistance, and also to Mr. D. Mrozek for editing the manuscript in English. A part of the present study was presented in June at the 10th International Congress of Endocrinology in San Francisco, USA. 


\section{References}

1. Zakarija M, McKenzie M, Banovac K (1980) Clinical significance of assay of thyroid-stimulating antibody in Graves' disease. Ann Intern Med 93: 2832.

2. Burman KD, Baker JR (1985) Immune mechanisms in Graves' disease. Endocr Rev 6: 183-231.

3. Smith BR, McLachlan SM, Furmaniak J (1988) Autoantibodies to the thyrotropin receptor. Endocr Rev 9: 106-121.

4. Volpé R (1991) Graves' disease: Pathogenesis. In: Braverman LE, Utiger RD (eds) Werner and Ingbar's the Thyroid: A Fundamental and Clinical Text. 6th ed. Philadelphia: J B Lippincott: 648-657.

5. Mori T, Akamizu T, Kosugi S, Sugawa H, Inoue D, Okuda J, Ueda Y (1994) Recent progress in TSH receptor studies with a new concept of 'Autoimmune TSH receptor disease'. Endocr J 41: 1-11.

6. Glinoer D, Hesh D, Lagasse R, Laurberg P (1987) The management of hyperthyroidism due to Graves' disease in Europe in 1986: Results of an international survey. Acta Endocrinol 115 (Suppl 285): 1-23.

7. Solomon B, Glinoer D, Lagasse R, Wartofsky L (1988) Management of hyperthyroidism due to Graves' disease: Results of a survey of members of the ATA. presented at the 63rd Meeting of the American Thyroid Association, Montreal, Canada.

8. The Japan Thyroid Association; Nagayama Y, Izumi M, Nagataki S (1989) The Management of hyperthyroidism due to Graves' disease in Japan in 1988. Encodrinol Japon 36: 299-314.

9. Hashizume K, Ichikawa K, Sakurai A, Suzuki S, Takeda T, Kobayashi M, Miyamoto T, Arai M, Nagasawa T (1991) Administration of thyroxine in treated Graves' disease: Effects on the level of antibodies to thyroid-stimulating hormone receptors and on the risk of recurrence of hyperthyroidism. $N$ Engl J Med 324: 947-953.

10. Edmonds C J, Tellez M (1994) Treatment of Graves' disease by carbimazole: High dose with thyroxine compared to titration dose. Eur J Endocrinol 131: 120-124.

11. Meng W, Niemeyer T, Kirsch G, Krabbe S, Schindler A (1995) Graves' disease - high dosed methimazole therapy and levothyroxine administration do not lead to better long time results. Thyroid 5: S106 (Abstract).

12. Hershman JM (1995) Editorial: Does thyroxine therapy prevent recurrence of Graves' hyperthyroidism? J Clin Endocrinol Metab 8: 14791480.

13. Tamai H, Hayaki I, Kawai K, Komaki G,
Matsubayashi S, Kuma K, Kumagai LF, Nagataki S (1995) Lack of effect of thyroxine administration on elevated thyroid stimulating hormone receptor antibody levels in treated Graves' disease patients. J Clin Endocrinol Metab 80: 1481-1484.

14. McIver B, Rae P, Beckett G, Wilkinson E, Gold A, Toft A (1996) Lack of effect of thyroxine in patients with Graves' hyperthyroidism who are treated with an antithyroid drug. $N$ Engl J Med 334: 220-224.

15. Rittmaster RS, Zwicker H, Abbott EC, Douglas R, Givner ML, Gupta MK, Lehman L, Reddy S, Salisbury SR, Shlossberg AH, Tan MH, York SE (1996) Effect of methimazole with or without exogenous l-thyroxine on serum concentrations of thyrotropin (TSH) receptor antibodies in patients with Graves' disease. J Clin Endocrinol Metab 81: 3283-3288.

16. Kuo S, Huang W, Hu C, Liao W, Fung T, Wu S (1994) Effect of thyroxine administration on serum thyrotropin receptor antibody and thyroglobulin levels in patients with Graves' hyperthyroidism during antithyroid drug therapy. Eur J Endocrinol 131: 125-130.

17. Weetman AP, McGregor AM, Hall R (1984) Evidence for an effect of antithyroid drugs on the natural history of Graves' disease. Clin Endocrinol (Oxf) 21: 163-172.

18. Laurberg P, Hansen PEB, Iversen E, Jensen SE, Weeke J (1986) Goitre size and outcome of medical treatment of Graves' disease. Acta Endocrinol 111: 39-43.

19. Young ET, Steel NR, Taylor JJ, Stephenson AM, Stratton A, Holcombe M, Kendall-Taylor P (1988) Prediction of remission after antithyroid drug treatment in Graves' disease. Quat J Med 66: 175189.

20. Reinwein D, Benker G, Lazarus JH, Alexander WD, the European multicenter study group on antithyroid drug treatment (1993) A prospective randomized trial of antithyroid drug dose in Graves' disease therapy. J Clin Endocrinol Metab 76: 1516-1521.

21. Mori T, Torizuka K (1973) Drug and radioiodide therapy in patients with Graves' disease. In: Yamada T, Ogata E, Nagataki S, Ochi Y, Torizuka K, Kumahara Y, Miyai K (eds) HYPERTHYROIDISM: Basic and Clinical Studies. Ishiyakushuppan, Tokyo: 109-130 (In Japanese).

22. Alexander WD, McLarty DG, Horton $P$, Pharmakiotis AD (1973) Sequential assessment during drug treatment of thyrotoxicosis. Clin Endocrinol 2: 43-50.

23. Mori T (1996) The management of hyperthyroidism. 
presented at the 10th International Congress of Endocrinology, San Francisco, US. June (manuscript in press).

24. Inoue $D$, Sato $K$, Enomoto $T$, Sugawa $H$, Maeda $M$, Inoko H, Tsuji K, Mori T, Imura H (1992) Correlation of HLA types and clinical findings in Japanese patients with hyperthyroid Graves' disease: Evidence indicating the existence of four subpopulations. Clin Endocrinol (Oxf) 36: 75-82.

25. Alexander WD, Harden RM, Koutras DA, Wayne E
(1965) Influence of iodine intake after treatment with antithyroid drugs. Lancet 2: 866-868.

26. Wartofsky L (1973) Low remission after therapy for Graves' disease: Possible relation of dietary iodine with antithyroid therapy results. JAMA 226: 1083-1088.

27. Solomon BL, Evaul JE, Burman KD, Wartofsky L (1987) Remission rates with antithyroid drug therapy: Continuing influence of iodine intake? Ann Intern Med 107: 510-512. 